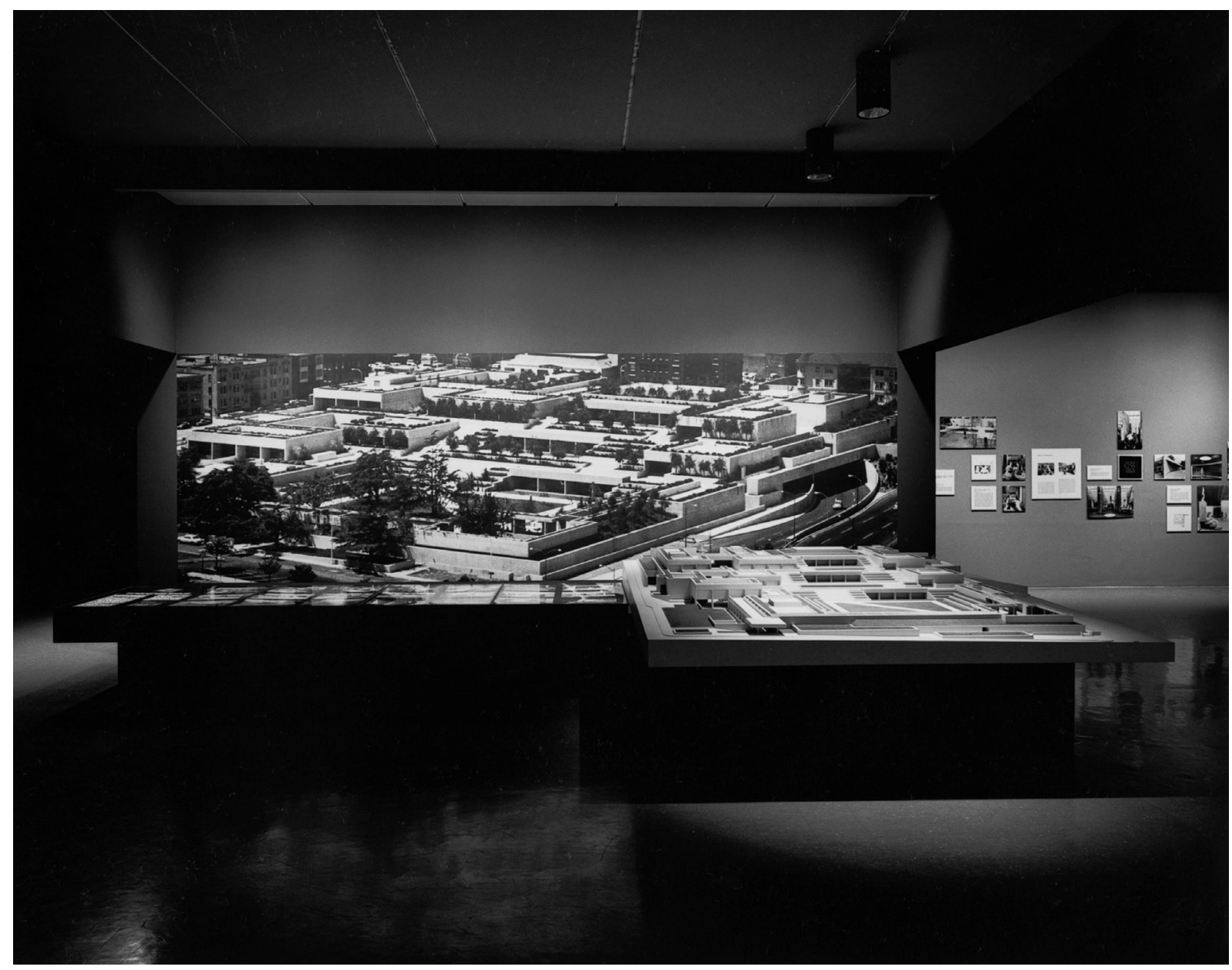




\section{3 exposiciones, 2 comisarios y 1 museo: Kevin Roche John Dinkeloo and Associates a través del MoMA, NYC Laura Sánchez}

Durante los años sesenta y setenta, el estudio formado por Kevin Roche John Dinkeloo and Associates gozó de una gran repercusión en el panorama arquitectónico internacional. Así lo constatan las tres exposiciones del MoMA en las que participaron y que son el hilo conductor de este artículo. Con un análisis pormenorizado de estas tres muestras se pretende demostrar el gran valor de las exposiciones de arquitectura como fuente de investigación ya que representan la actualidad y sirven como termómetro de las discusiones del momento. El objetivo es establecer el marco teórico en el que el museo inserta el trabajo de KRJDA y la manera en la que relaciona a este equipo con los debates y profesionales del momento.

\section{PALABRAS CLAVE}

Estados Unidos, comisariado, arquitectura moderna, Arthur Drexler, Ludwig Glaeser KEYWORDS

United States of America, Curatorship, Modern Architecture, Arthur Drexler, Ludwig Glaeser

En el momento en el que las obras de arquitectura y sus autores pasaron a ser considerados objetos dignos de ser estudiados y expuestos en los museos, el discurso arquitectónico adquirió nuevas aspiraciones de relevancia cultural, extendiéndose más allá de la propia profesión. La institución que mayor influencia ha tenido en este sentido es, sin duda, el Museo de Arte Moderno de Nueva York (MoMA). Muchas de sus exposiciones abrieron caminos a la arquitectura desde que en 1932, y a cargo de Philip Johnson, se inaugurase el Departamento de Comisariado de Exposiciones de Arquitectura y Diseño con la exposición "Modern Architecture: International Exhibition". Los años sesenta y setenta, en los que se centra este artículo, fueron de gran actividad para el departamento, que organizó variadas exposiciones, intercalando muestras colectivas organizadas en base a ideas o tipologías con retrospectivas sobre un único arquitecto.

\begin{abstract}
Laura Sánchez Carrasco
Arquitecta por la ETSAM desde 2007 y Doctora en Arquitectura por la UPM desde 2017. En la actualidad es profesora asociada en el Departamento de Composición Arquitectónica de la ETSAM, donde imparte diferentes asignaturas. Sus investigaciones, difundidas mediante artículos y congresos, se centran en la arquitectura de los Estados Unidos tras la II Guerra Mundial, concretamente en el trabajo de Kevin Roche John Dinkeloo and Associates. También ha sido miembro del equipo investigador de varios proyectos de investigación de financiación estatal. Compagina la labor docente e investigadora con la práctica profesional y ha sido premiada en distintos concursos de arquitectura.
\end{abstract}

Fig. 01

Maqueta y fotografía del Museo de Oakland en la exposición "Architecture of Museums" en el MoMA (1968). Photographic Archive. The Museum of Modern Art Archives, New York. IN867.5. Photograph by George Cserna. 
Estas dos décadas fueron también los años de mayor repercusión de Kevin Roche John Dinkeloo and Associates (KRJDA) en el panorama arquitectónico internacional, y su presencia en el MoMA entre 1966 y 1981 así lo constata'. Un total de tres exposiciones, organizadas por dos comisarios diferentes en un museo que durante esos quince años destacó a otras grandes figuras como James Stirling, Frei Otto, Charles Eames, Louis Kahn, Mies van der Rohe, Le Corbusier o Marcel Breuer².

En este texto se pretende demostrar el gran valor de las exposiciones como fuente de investigación para entender el discurso teórico que sostiene la obra de un arquitecto y además, insertarlo y posicionarlo correctamente en el momento en el que fue desarrollado. Prácticamente la totalidad de la documentación utilizada se ha extraído del archivo del propio museo. Por un lado el archivo digital, abierto y accesible por internet, que ofrece las notas de prensa, fotografías y, en algún caso, catálogos; y por otro lado los documentos que conserva el museo en su sede, que amplían la información a todo tipo de folletos publicitarios, definiciones del montaje de la propia exposición, correspondencia entre comisarios y autores, entrevistas, además de información variada que ayuda a entender la gestación, el proceso y los intereses de cada muestra. Estudiando estos documentos se han podido extraer los conceptos que los comisarios desatacaban de la obra de KRJDA. Unas ideas que enlazaban sin duda alguna con las inquietudes del momento y que, en gran medida, fueron el contacto de estos arquitectos con el gran público.

La primera cuestión a valorar es la variedad de las exposiciones a las que se va a hacer referencia en el artículo y que se resume en la siguiente tabla (fig. 02). La primera de ellas tuvo lugar en 1968 y fue de carácter colectivo. Estaba enfocada al estudio tipológico, en este caso, de los museos, un campo en el que KRJDA destacaron desde el principio de su carrera.

La segunda muestra, de 1970, se entiende mejor como un monográfico de la arquitectura estadounidense del momento, reflejada en tres grandes figuras americanas: Roche, Rudolph y Johnson. Un poco más tarde, en 1979, una tercera exposición, de nuevo colectiva, que indaga en cuestiones de estilo y teoría de la arquitectura. En ella se incluía a muchos arquitectos y pretendía demostrar las conexiones entre la arquitectura de ese momento con los inicios de la modernidad. El estudio pormenorizado de estas tres exposiciones descubre un marco teórico en el que se sustenta la obra de KRJDA.

El 25 de septiembre de 1968 abría al público la exposición "Architecture of Museums", comisariada por Ludwig Glaeser. Durante casi dos meses (se clausuró el 11 de noviembre del mismo año) se expusieron maquetas, murales fotográficos y demás documentación gráfica de 71 museos. Aunque la mayoría de estas piezas se habían construido entre los años cincuenta y sesenta, también se incluían algunos ejemplos históricos e incluso proyectos no construidos de un total de veintidós nacionalidades.

Glaeser consideró adecuado incorporar a esta exposición el Museo de Oakland, a pesar de que aún faltaban unos meses para estar completamente terminado, y dos proyectos más de KRJDA que nunca se llegaron a construir: el estructuralmente arriesgado Air Force Museum (1964) y la pequeña Orangerie (1968) que se diseñó para el jardín de uno de sus clientes ${ }^{3}$. Pero fue el museo californiano el que recibió las mayores atenciones y sobre el que se produjo un debate más intenso, apoyado también por el despliegue documental que de él se hacía y que consistía 


\begin{tabular}{|c|c|c|c|}
\hline $\begin{array}{l}\text { Título de la exposición } \\
\text { (Exhibition title) } \cdot \text { Fecha } \\
\text { (Date) } \cdot \text { Comisario } \\
\text { (Curator) }\end{array}$ & Enfoque (Approach) & Objetivos (Goals) & $\begin{array}{l}\text { Obras incluidas de } \\
\text { KRJDA (KRJDA's works } \\
\text { included) }\end{array}$ \\
\hline $\begin{array}{l}\text { Architecture of } \\
\text { Museums } \\
1968 \\
\text { Ludwig Glaeser }\end{array}$ & $\begin{array}{l}\text { Tipológico } \cdot \text { Museos } \\
(\text { Typological } \cdot \text { Museums) }\end{array}$ & $\begin{array}{l}\text { Mostrar la evolución } \\
\text { tipológica y formal de los } \\
\text { museos } \cdot \text { Definir cuál es la } \\
\text { función del museo } \\
\text { (Showing the typological } \\
\text { and formal evolution of } \\
\text { museums } \cdot \text { Defining what } \\
\text { the museum's function is) }\end{array}$ & $\begin{array}{l}\text { - Oakland Museum } \\
\text { - Air Force Museum } \\
\text { - Orangerie }\end{array}$ \\
\hline $\begin{array}{l}\text { Work in Progress: } \\
\text { Architecture by Philip } \\
\text { Johnson, Kevin Roche, } \\
\text { Paul Rudolph } \\
1970 \\
\text { Arthur Drexler }\end{array}$ & $\begin{array}{l}\text { Monográfico } \cdot \text { El autor y } \\
\text { su obra } \\
\text { (Monographic } \cdot \text { Author } \\
\text { and its work) }\end{array}$ & $\begin{array}{l}\text { Divulgar el alto nivel de la } \\
\text { arquitectura de los EEUU } \\
\text { en esos años a través de } \\
\text { arquitectos en activo que } \\
\text { hayan contribuido a } \\
\text { conseguir esa situación } \\
\text { relevante · Mostrar la } \\
\text { condición de la } \\
\text { arquitectura como objeto } \\
\text { artístico } \\
\text { (Disseminate the high } \\
\text { level of US architecture in } \\
\text { those years through } \\
\text { professionals who have } \\
\text { contributed to achieve that } \\
\text { relevant situation · } \\
\text { Showing architecture as an } \\
\text { artistic object ) }\end{array}$ & $\begin{array}{l}\text { - Irwin Union Bank } \\
\text { - UN Plaza } \\
\text { - IBM Museum } \\
\text { - Cummins Engine Co. } \\
\text { (Walesboro) } \\
\text { - College Life Insurance } \\
\text { - Knights of Columbus } \\
\text { - New Haven Coliseum } \\
\text { - Rochester I.T. }\end{array}$ \\
\hline $\begin{array}{l}\text { Transformations in } \\
\text { Modern Architecture } \\
1979 \\
\text { Arthur Drexler }\end{array}$ & $\begin{array}{l}\text { Teórico } \cdot \text { Evolución de la } \\
\text { forma arquitectónica } \\
\text { desde la modernidad } \\
\text { (Theoretical } \text { Evolution of } \\
\text { architectural form since } \\
\text { modernity) }\end{array}$ & $\begin{array}{l}\text { Demostrar que la } \\
\text { arquitectura de los años } \\
\text { sesenta y setenta es una } \\
\text { evolución de los conceptos } \\
\text { de la arquitectura moderna } \\
\text { de los años veinte y treinta } \\
\text { (Showing that the } \\
\text { architecture of the } 60 \text { 's } \\
\text { and } 70 \text { 's is an evolution of } \\
\text { the concepts of modern } \\
\text { architecture of the } 20 \text { 's } \\
\text { and } 30 \text { 's) }\end{array}$ & $\begin{array}{l}\text { - Knights of Columbus } \\
\text { - College Life Insurance } \\
\text { - Ford Foundation } \\
\text { - Oakland Museum }\end{array}$ \\
\hline
\end{tabular}

02

en una gran maqueta, una fotografía de tamaño mural y diferentes dibujos e informaciones colocadas en un expositor horizontal.

El objetivo de esta exposición consistía, no sólo en la divulgación de estas piezas dedicadas al arte, sino en apoyar el debate existente en aquellos momentos sobre qué función debían tener los museos en la sociedad 4 . El comisario afirmaba que los museos elegidos, además de ser excelentes en su arquitectura "sugerían un ambiente agradable, acorde a los valores inmanentes de la colección y los momentos contemplativos del visitante"s.

Se iniciaba la exposición en el s. XVIII con el Cenotafio de Newton de Boullée (1783) y continuaba con algunas obras de Karl Friederich Schinkel como el Altes Museum (1830) para centrarse después en grandes piezas de los cincuenta y sesenta. De estos años se expusieron edificios míticos de Marcel Breuer, Louis I. Kahn, Alvar Aalto, Oscar Niemeyer o Aldo van Eyck. Entre los ejemplos coetáneos al Museo de Oakland, el comisario destacaba el Museo de Arte en Siracusa (1968) de I. M. Pei, el Museo Joseph I. Nirshhorn en Washington de Gordon Bunshaft (aunque no se terminaría hasta 1974) o las actuaciones que Michael Graves había desarrollado en el Museo Newark desde finales de los años sesenta.
Fig. 02

Tabla resumen de las exposiciones analizadas, incluyendo los proyectos de KRJDA incorporados a las mismas. Realizada por la autora. 
Fig. 03

Espacio dedicado al Museo de Oakland, con la Neue Nationalgalerie de Mies ven der Rohe en Berlín y el Museo Guggenheim de Nueva York de Frank Lloyd Wright al fondo. Dentro de la exposición "Architecture of Museums" en el MoMA. Photographic Archive. The Museum of Modern Art Archives, New York. IN867.5. Photograph by George Cserna.
El Museo de Oakland pertenecía al conjunto de los llamados museos 'invisibles' o enterrados, entre los que también se incluían el museo Shrine of the Book en Jerusalén (1965) de Frederick Kiesler y Armand Jartos, la Galería de Arte en New Canaan (1965) de Philip Johnson, el lapidario Greco-Romano de Buzenol-Montauban en Bélgica (1960) de Constantin L. Brodzki, la galería de arte Albright-Knox (1962) de SOM, el museo Louisiana de Copenhague (1958) de Jørgen Bo y Vilhelm Wohlert y, aunque dentro de un contexto completamente distinto, el Museo del Tesoro en la Catedral de San Lorenzo de Génova (1956) de Franco Albini6.

En la nota de prensa enviada por el MoMA a los medios, de cuatro páginas, se mencionaba al Museo de Oakland en un párrafo de diez líneas, destacándose así entre el resto de obras expuestas. Sólo los comentarios sobre Mies van der Rohe, Le Corbusier y Frank Lloyd Wright tienen una extensión similar en este texto. Además de una breve descripción, Ludwig Glaeser emite una valoración positiva sobre el proyecto de KRJDA, del que destaca la innovación tipológica del edificio y su habilidad para integrarse en el entorno:

\footnotetext{
"Oakland no es sólo un esquema de museo excepcional sino también una solución arquitectónica única. En lugar de esforzarse en diseñar un monumento a la cultura, los arquitectos han enterrado el edificio bajo su propio paisaje. El edificio así reconoce su función urbana al ser en realidad un parque, pero también reconoce su función ampliada como museo al ofrecer un lugar de congregación"7.
}

La relevancia que este museo adquirió en la exposición también tuvo su reflejo en las reseñas que la prensa escrita dedicó a la muestra. Es el caso del artículo de Ada Louise Huxtable para The New York Times del 25 de septiembre de 1968. Bajo el título "Architecture: A Museum is also Art, Exhibition Shows" se acompaña de una imagen del Museo de Oakland, al que destaca por su carácter innovador

\footnotetext{
"Desde el punto de vista del diseño y el entorno, Oakland puede ser uno de los edificios más seriamente revolucionarios en el mundo"9.
}

Otro de los aspectos que favorecieron esa posición tan destacada del museo californiano en esta exposición fue su situación intermedia entre las dos corrientes que polarizaban la muestra: aquéllos museos que se mostraban como un contenedor neutro que otorgaba todo el protagonismo a las obras de arte expuestas en ellos (como la galería berlinesa de Mies van der Rohe) o aquéllos que se consideraban una obra de arte en sí mismos (con el Guggenheim de Nueva York como máximo representante) ${ }^{10}$. El ejemplo de Oakland disfrutaba de una admirada conciliación entre esos dos aspectos. Su carácter paisajístico, aterrazado y novedoso hacían del museo un gran objeto que contemplar pero a su vez, la neutralidad de los espacios interiores permitía a los visitantes disfrutar de las obras expuestas

En 1970, sólo cuatro años después de la formación oficial de KRJDA, el MoMA organizó una retrospectiva dedicada a tres arquitectos en activo: Philip Johnson, Kevin Roche y Paul Rudolph. En esta ocasión, el comisario fue Arthur Drexler, que se encontraba a cargo del departamento de arquitectura y diseño desde el año anterior. Drexler 

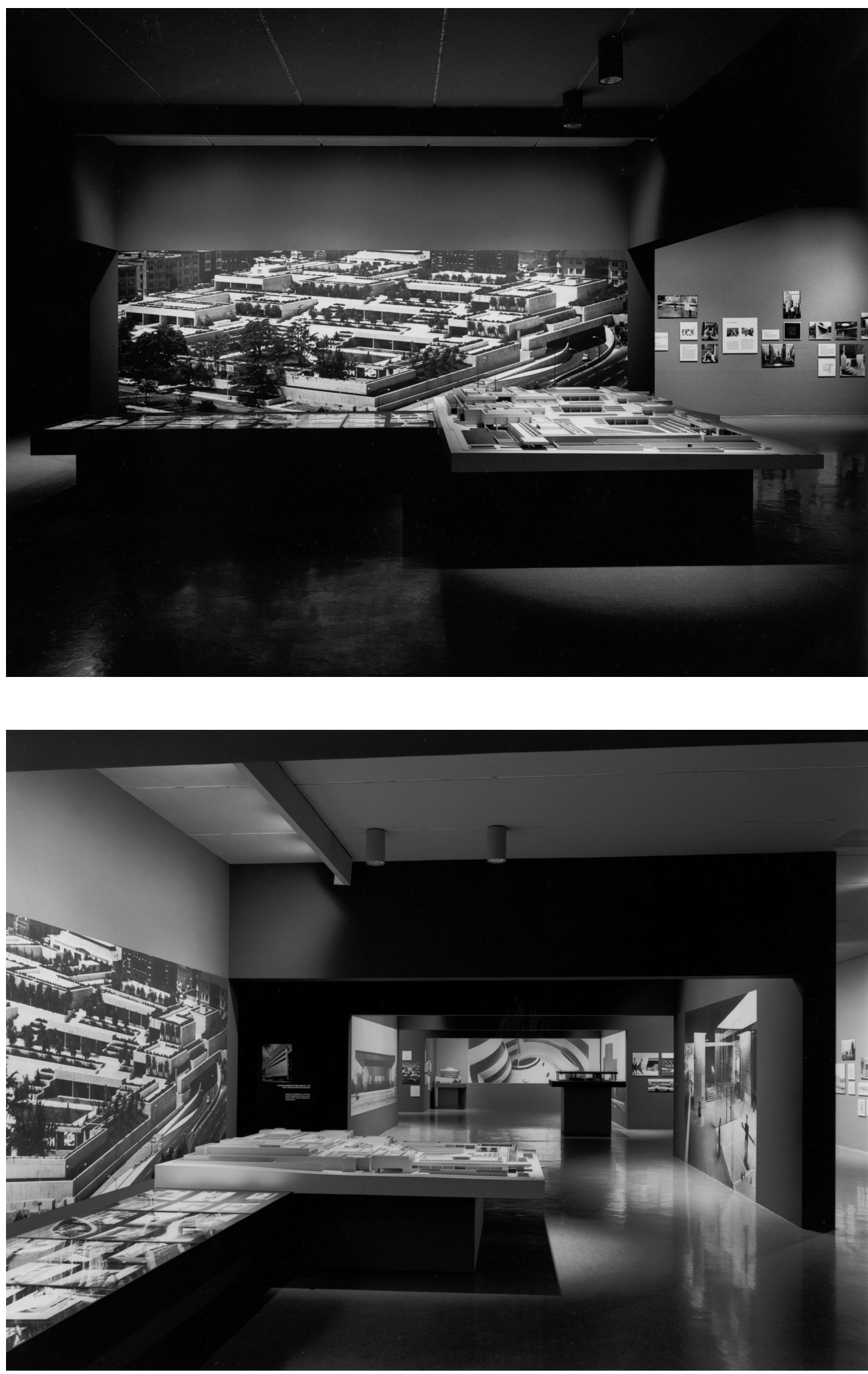


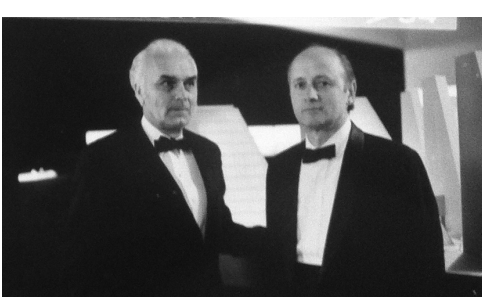

04

Fig. 04

John Dinkeloo y Kevin Roche delante de la maqueta del College Life Insurance en la cena organizada con motivo de la exposición del MoMA "Work in Progress: Architecture by Philip Johnson, Kevin Roche, Paul Rudolph". Imagen procedente de: The Museum of Modern Art Archives, NY. Collection: PI. Series.Folder: II.B.829. seleccionó un total de veinticinco proyectos que se mostraron al público entre el uno de octubre de 1970 y el tres de enero de 1971, mediante maquetas, dibujos y fotografías

La exposición contenía principalmente proyectos aún sin construir que, a criterio del comisario, reflejaban un compromiso con la idea de que la arquitectura debe producir un objeto artístico". Arthur Drexler pretendía contar al público el nivel de excelencia que había adquirido la arquitectura norteamericana en esos años, un nivel que él consideraba superior al de cualquier otro lugar del mundo. En ese sentido, apostó por tres arquitectos que, según su criterio, habían hecho grandes contribuciones a esta escena americana.

\footnotetext{
"Esta exposición repasa veinticinco proyectos de tres arquitectos que han hecho grandes contribuciones a la escena americana. (...) Todos ellos reflejan un compromiso con la idea de que la arquitectura, además de ser tecnología, sociología y filosofía moral, debe producir, en última instancia, obras de arte, si merece la pena preocuparse por eso"12.
}

Como se ha comentado, muchos de estos proyectos aún estaban en construcción o en fases iniciales de diseño, circunstancia que se reflejaba en el título de la exposición, "Work in Progress: Architecture by Philip Johnson, Kevin Roche, Paul Rudolph"13.

Los objetivos de Arthur Drexler en este caso y su interés en cada uno de los participantes, se entienden bien en la primera pregunta que el comisario le hace a Kevin Roche en una entrevista previa que hace a todos los autores con el objetivo de comprender el pensamiento de estos tres arquitectos:

\footnotetext{
"Permíteme que rápidamente te dé una idea del tipo de preguntas que les formulé a Philip y Paul. Respecto a Philip yo estaba particularmente interesado en su actitud hacia la edificación actual, porque la mayoría de su trabajo ha estado afectado por la articulación y algunas veces por la decoración del edificio y yo quería que hablase sobre esto y el modo en que él lo considera ahora, y con Paul yo estaba de nuevo interesado, no tanto en el edificio como en problemas formales comparables. Estoy más interesado en extraer de cada uno de vosotros actitudes sobre el arte de la arquitectura, no necesariamente sobre los problemas del mundo, excepto que me interesaban las reacciones de Philip y Paul, y creo que tuyas también, sobre qué esta ocurriendo en las escuelas ahora y tus propias sensaciones sobre cómo enseñarías arquitectura si de repente te encontrases en esa incómoda posición"14.
}

Los motivos por los que Arthur Drexler incluía a KRJDA en la muestra los aclaraba el comisario en los textos que acompañaban a las obras expuestas:

"El trabajo de Kevin Roche y John Dinkeloo puede parecer a primera vista un sencillo e inusualmente preciso manejo de la construcción convencional en hierro y vidrio. Pero Roche se ha ido interesando cada vez más en problemas de escala urbana, ha buscado agrandar el tamaño aparente de los edificios urbanos, para que pudieran estabilizar visualmente sus alrededores y que se reconozcan a gran distancia. Donde la mayoría de los arquitectos se esforzarían por tener un mayor rango de dimensiones -desde la más pequeña 
textura visible a los grandes elementos- Roche tiende a agrandar la escala en todas partes, como con las cuatro torres de esquina del edificio de oficinas Knight of Columbus en New Haven, produciendo una especie de orden gigante que es asombrosamente eficaz en el entorno urbano"15.

Es decir, la primera idea importante que se manejaba tenía que ver con la escala entendida desde el entorno urbano y la presencia de los edificios en el mismo. Un trabajo que proponía la eficacia de las grandes formas en todos los niveles.

En segundo lugar, Drexler destacaba las cuestiones técnicas de los edificios de KRJDA y, en concreto, el trabajo y la investigación con el vidrio que les permitía incluir jardines dentro del edificio para cualificar los espacios interiores, una característica de su trabajo que han continuado desarrollando a lo largo de su carrera.

"Otra característica del trabajo de Roche es el edificio concebido casi en su totalidad como un envoltorio de vidrio -tanto la cubierta como las fachadas- usando el vidrio para hacer más evidentes los, a menudo, espacios gigantescos -algunas veces más como invernaderos que como espacios convencionales- que ahora pretende incorporar en la mayoría de sus grandes edificios. Así, el jardín interior de la Fundación Ford en la calle 42 ha evolucionado hacia un espacio vertical envuelto en vidrio de cuarenta pisos, en el proyecto de desarrollo de las Naciones Unidas"16.
Fig. 05

Maquetas y fotografías del trabajo de KRJDA expuesto en el MoMA en 1970. Las maquetas destacan la transparencia y el uso del vidrio del UN Plaza y el College Life Insurance. Photographic Archive. The Museum of Modern Art Archives, New York. IN940.2. Photograph by Alexandre Georges.

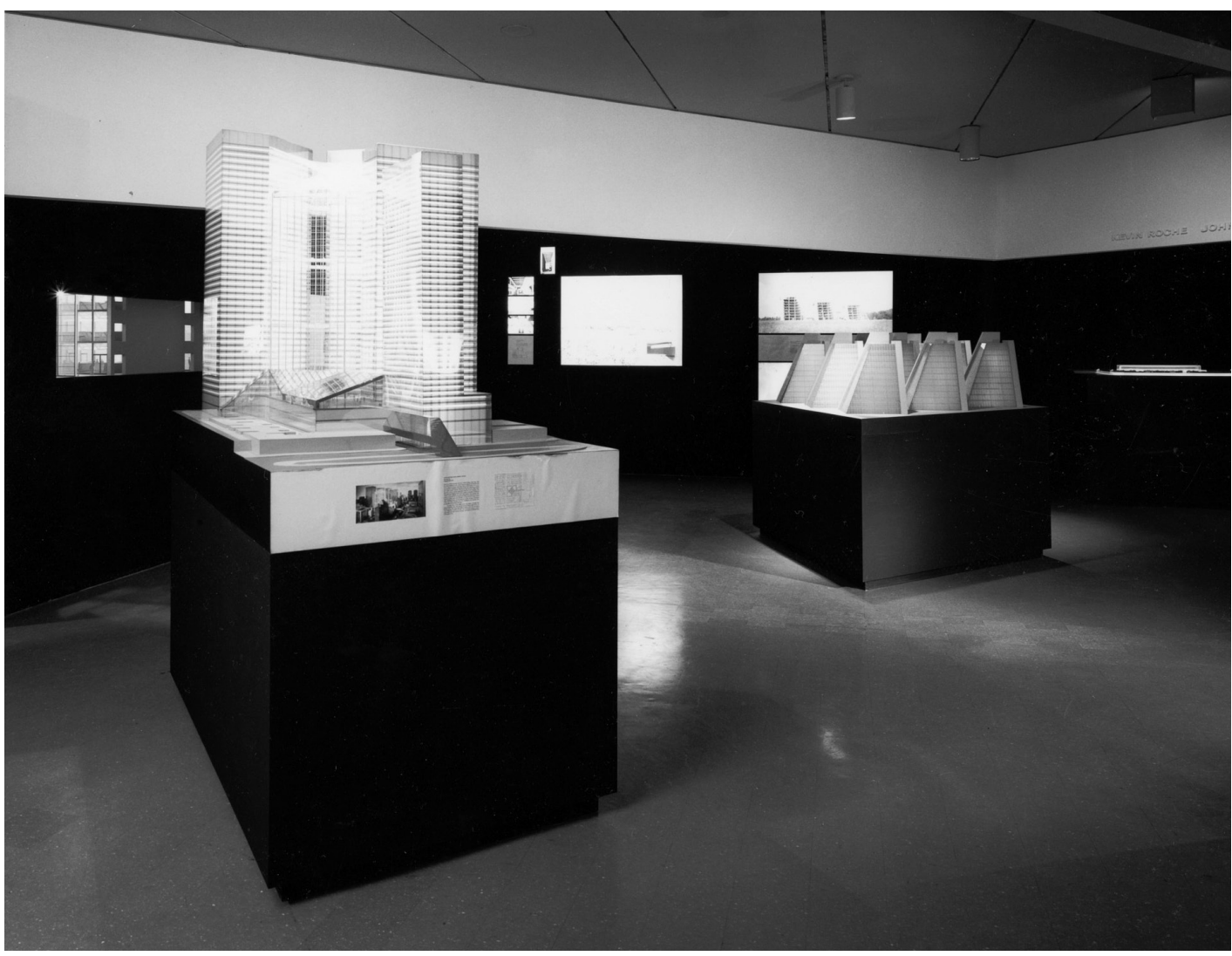




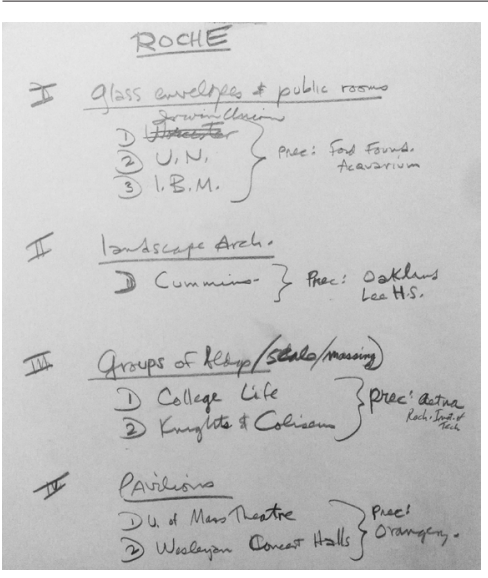

06

Fig. 06

Documento de preparación de la exposición en el que se indican los cuatro conceptos en los que Arthur Drexler dividía los proyectos de KRJDA. El último, pabellones, se desestimó finalmente. Imagen procedente de: The Museum of Modern Art Archives. Collection: MoMA Exhs. Series.Folder: 940.18.
También destacaba Drexler las cuestiones paisajísticas del trabajo de KRJDA, que estaban representadas por dos proyectos de gran extensión en el territorio y siempre alabados por su manera de integrarse en él: el anteriormente citado museo de Oakland y la fábrica para la Cummins Engine Company en Walesboro, Columbus.

\footnotetext{
"A pesar de su preocupación por las formas arriesgadas y llamativas, Roche ha emprendido ocasionalmente un objetivo más relacionado con el diseño de paisaje que con la propia arquitectura. Su museo de Oakland en California es esencialmente un aterrazamiento del lugar; su diseño reciente para la planta manufacturera para la Cummins Engine Company en Indiana ubica la mayoría del edificio bajo tierra, de manera que aparece como una ligera y relativamente discreta interrupción del paisaje llano"17.
}

Las propuestas de Roche, continúa enfatizando Drexler, son valiosas por utilizar herramientas conocidas o tradicionales provocando situaciones inesperadas.

\footnotetext{
"Muchos de los edificios de Roche presentan detalles o espacios suficientemente familiares en otros contextos, pero en sus interpretaciones adoptan un trasfondo casi surrealista. La moderación y sobriedad con la que se fijan estos efectos no disfrazan la percepción que subyace en Roche de lo fantástico en la vida urbana del siglo veinte"18.
}

En la exposición se mostraban nueve proyectos de Philip Johnson (dos de ellos construidos), ocho de KRJDA, de los cuales se habían terminado cuatro, y seis proyectos de Paul Rudolph, todos inacabados. En total había catorce maquetas, seis de Johnson, cinco de Roche y Dinkeloo y tres de Rudolph ${ }^{19}$. Lógicamente, por su condición de monográfica, esta exposición es la que desarrolla una investigación más exhaustiva de la obra de KRJDA y de la que más carga teórica se puede extraer. Como se ha podido observar, las alusiones y los conceptos valorados son muchos.

Los proyectos de KRJDA incluidos en "Work in Progress" eran el Irwin Union Bank \& Trust Company en Columbus, el United Nations Development Center en Nueva York, el Computer Technology Museum en Armonk, la Cummins Engine Company Manufacturing Plant en Columbus, el edificio para el College Life Insurance Company of America en Indianapolis, la torre Knights of Columbus Office Building en New Haven, el Coliseum en New Haven y el nuevo campus del Rochester Institute of Technology. De los cinco primeros de la lista, aportaron maquetas a la exposición, algunas de las cuales se hicieron específicamente para la ocasión y se enfocaron en transmitir esa idea de envoltura transparente y tecnológica de vidrio, como la del edificio College Life Insurance ${ }^{20}$. Los cuatro últimos eran proyectos ya construidos.

La figura 07 documenta la relación de los proyectos elegidos (junto a los considerados precedentes en cada categoría) y su distribución según las ideas expuestas anteriormente. El primer grupo englobaba las envolturas de vidrio y los espacios públicos que se creaban en su interior. En este apartado se incluía el Irwin Union Bank (que sustituía al Worcester Bank que se había elegido inicialmente), el United Nations Development y el Computer Technology Museum, nombrando como precedentes la Fundación Ford y el Acquarium de Washington, un 


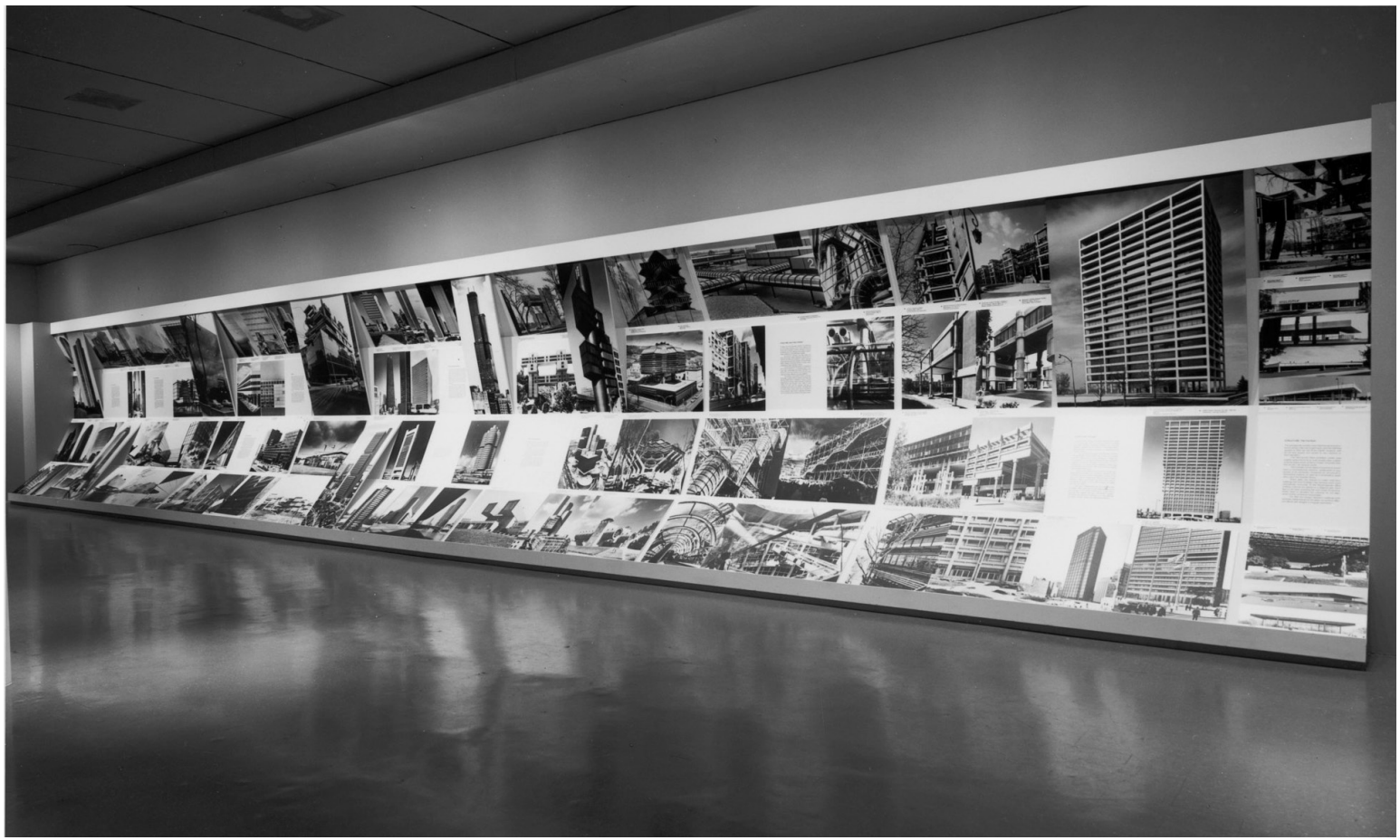

07

museo en colaboración con Charles Eames que nunca llegó a construirse. La segunda categoría se centraba en el paisaje, y proponía el proyecto para la fábrica de la Cummins Engine Company en Columbus. El precedente era el Museo de Oakland y, por su horizontalidad, el instituto Richard C. Lee en New Haven. La última sección pretendía destacar la agrupación de elementos, haciendo referencia al uso de la masa y la escala. En este apartado se incluyeron el College Life Insurance de Indianapolis, el conjunto de la torre Knights of Columbus y el Coliseum en New Haven y el Rochester Institute of Technology, realizado en colaboración con otros arquitectos. En un principio se pensaba incluir una cuarta categoría dedicada a pabellones, que mostraría el Teatro de la Universidad de Massachusetts y el Concert Hall de la Universidad de Wesleyan, con el precedente de la pequeña Orangerie. Finalmente, esta categoría se desestimó ${ }^{1}$.

En las entrevistas previas a la exposición, el comisario también dejaba claro que le interesaba la arquitectura entendida como una expresión artística, una posición de la que Roche siempre se había mantenido distante. Roche argumentaba que aceptaba la arquitectura como un arte, tal y como evidenciaba la historia, pero matizaba que plantear la arquitectura sólo desde la dimensión artística era no entender su verdadera naturaleza. Su discurso se mantenía más pragmático y asumía que el mayor reto de la arquitectura en esos años era acomodar a un gran número de personas de manera que se las pudiera ofrecer la oportunidad de vivir una vida de calidad superior ${ }^{22}$. Para Roche la mayor relación de la arquitectura y el arte se basaba en que en ambos casos, se pretendía expresar las condiciones del momento en el que se realizaban y ofrecer soluciones.
Fig. 07

Mural fotográfico de la exposición Transformation in Modern Architecture (1979). En la parte central se puede observar la torre Knights of Columbus de KRJDA en New Haven. 


\begin{abstract}
"Supongo que en el pasado los pintores pintaban porque había una razón para pintar, algo y por encima de lo que sentían que tenían que hacer -había realmente una razón social y desarrollaban sus técnicas y expresaban sus habilidades artísticas o capacidades dentro de esa razón. $Y$ yo creo que ése es un acercamiento muy legítimo para el problema de la

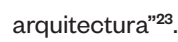

La última exposición a analizar tuvo lugar entre el 23 de febrero de 1979 y el 24 de abril del mismo año. Arthur Drexler, aún encargado del departamento de arquitectura y diseño del MoMA, comisariaba "Transformations in Modern Architecture". Una muestra de carácter coral que exponía fotografías de más de cuatrocientos edificios y que ese mismo año se convertiría en un libro de gran éxito editado por el propio museo.

La exposición reivindicaba que la historia de la arquitectura moderna entre los años sesenta y setenta suponía una elaboración de las ideas pronunciadas por primera vez en los años veinte y treinta ${ }^{24}$. Obviamente, al tratarse de una investigación que incluía a tantos autores, las alusiones a Roche y Dinkeloo eran pocas, pero sí resulta de gran interés cómo fueron incluidos y con qué otros proyectos y arquitectos se les relacionaba.

Se establecieron tres categorías: arquitectura como invención de la forma escultórica, arquitectura como forma estructural y arquitectura vernácula. El trabajo de KRJDA sólo se incluye en la segunda categoría que, desde el museo, se definía de la siguiente manera:

\footnotetext{
"La forma estructural trabaja con lo que el arquitecto Mies van der Rohe llamaba arquitectura de 'piel y huesos': un esqueleto estructural de acero u hormigón que está cubierto por una piel metálica o de vidrio. Este tipo de arquitectura confía en la estructura para comunicar información visual sobre un edificio, independientemente del uso al que está destinado. El desarrollo actual de este tipo de arquitectura vuelve al énfasis inicial sobre la piel, como se puede ver en los ubicuos edificios 'espejo' de hoy en día. Utilizando vidrio tintado y reflectante, comunican poca o ninguna información sobre ellos mismos y llevan la abstracción arquitectónica hasta su punto álgido. Una sección especial de esta exposición es un espacio dedicado a las transparencias de color de estos edificios especulares, en los que 'la sustancia se desmaterializa y la técnica objetiva culmina en la contemplación subjetiva de las nubes y la luz del sol'”25.
}

A tenor de la definición de esta segunda categoría, los conceptos que avalaban la obra de KRJDA para Arthur Drexler tenían que ver con las relaciones entre la estructura y la piel, el desarrollo de las envolventes de vidrio y su consecuente abstracción formal. Esta idea es muy cercana a las inquietudes que exhibían los propios arquitectos en sus comentarios y en sus edificios. Conviene recordar el interés de John Dinkeloo en la mejora de la industria de la construcción y, concretamente, en el desarrollo de nuevos materiales. El vidrio especular, tan presente en esta exposición, fue precisamente una de las mayores aportaciones de Dinkeloo a la historia de la arquitectura.

Pero como se ha demostrado a lo largo del artículo, esta afición por el desarrollo de la técnica no ha sido la única inquietud teórica 
que el MoMA ha asignado a las obras de KRJDA, o mejor dicho, a su discurso. Por el contrario, el museo neoyorquino ha ayudado a establecer paralelismos con distintas obras y arquitectos coetáneos a Roche y Dinkeloo y les ha nutrido de un sustento teórico que se sintetiza en la siguiente tabla.

A modo de resumen, mediante estas tres exposiciones se podrían establecer tres grandes bloques de ideas asociadas a KRJDA: la innovación tipológica, reflejada en el novedoso Museo de Oakland y la creación de espacios verdes interiores; la innovaciones técnicas y materiales, con especial atención en los múltiples desarrollos del vidrio y, por último, la cuidada relación de su obra con el paisaje y el entorno. Todos ellos, temas de plena actualidad en los años de estudio que corroboran la relevancia y el papel predominante de estos arquitectos en el panorama arquitectónico estadounidense desde el inicio de su trayectoria profesional. RA

\begin{tabular}{|c|c|c|}
\hline $\begin{array}{l}\text { Título de la exposición } \\
\text { (Exhibition title) } \cdot \text { Fecha } \\
(\text { Date }) \cdot \text { Comisario } \\
(\text { Curator })\end{array}$ & $\begin{array}{l}\text { Ideas extraídas sobre el trabajo expuesto de } \\
\text { KRJDA (Ideas extracted on the exposed } \\
\text { work of KRJDA) }\end{array}$ & $\begin{array}{l}\text { Obras relacionadas con el } \\
\text { trabajo de KRJDA } \\
\text { arquitectos (Buildings related } \\
\text { to KRJDA's works) }\end{array}$ \\
\hline $\begin{array}{l}\text { Architecture of } \\
\text { Museums } \\
1968 \\
\text { Ludwig Glaeser }\end{array}$ & $\begin{array}{l}\text { Relación con el entorno: museo enterrado } \\
\text { (Connection with the surroundings: buried } \\
\text { museum) } \\
\text { Innovación tipológica: conexión de } \\
\text { espacios de exposición y espacios abiertos, } \\
\text { configuración aterrazada } \\
\text { (Typological innovation: connection } \\
\text { between exhibition spaces and open spaces, } \\
\text { terraced configuration) }\end{array}$ & $\begin{array}{l}\text { - Shrine of the Book } \\
\text { (Frederick Kiesler / Armand } \\
\text { Jartos) } \\
\text { - New Canaan Gallery (Philip } \\
\text { Johnson) } \\
\text { - Gallo-Roman Lapidary } \\
\text { (Constantin L. Brodzki) } \\
\text { - Museum of the Treasury at } \\
\text { San Lorenzo Cathedral } \\
\text { (Franco Albini) }\end{array}$ \\
\hline $\begin{array}{l}\text { Work in Progress: } \\
\text { Architecture by Philip } \\
\text { Johnson, Kevin Roche, } \\
\text { Paul Rudolph } \\
1970 \\
\text { Arthur Drexler }\end{array}$ & $\begin{array}{l}\text { Materialidad y envolturas de vidrio } \\
\text { (Materiality and glass enclosures) } \\
\text { Arquitectura y paisaje } \\
\text { (Architecture and landscape) } \\
\text { Organización de elementos en un } \\
\text { conjunto } \\
\text { (Grouping elements in a complex) } \\
\text { Arquitectura y arte } \\
\text { (Architecture and art) } \\
\text { Escala - masa - solidez } \\
\text { (Scale - mass - solidity) } \\
\text { 'Greenhouses' }\end{array}$ & $\begin{array}{l}\text { No se establecen paralelismos } \\
\text { entre obras concretas de los } \\
\text { tres arquitectos } \\
\text { (No parallels are drawn } \\
\text { between the works of the } \\
\text { three architects) }\end{array}$ \\
\hline $\begin{array}{l}\text { Transformations in } \\
\text { Modern Architecture } \\
1979 \\
\text { Arthur Drexler }\end{array}$ & $\begin{array}{l}\text { Forma estructural: dicotomía entre la piel y } \\
\text { la estructura del edificio } \\
\text { (Structural form: dichotomy between the } \\
\text { skin and the structure of the building) }\end{array}$ & $\begin{array}{l}\text { No se establecen paralelismos } \\
\text { concretos pero se engloba a } \\
\text { KRJDA dentro de los } \\
\text { interesados por la forma } \\
\text { estructural } \\
\text { (No parallels with other } \\
\text { buildings are drawn but } \\
\text { KRJDA is included among } \\
\text { those interested in structural } \\
\text { form) }\end{array}$ \\
\hline
\end{tabular}

Fig. 08

Tabla resumen de los conceptos asociados a KRJDA en cada una de las exposiciones y de las relaciones que estas muestras establecían entre los protagonistas del artículo y otros profesionales u obras. Realizada por la autora. 


\section{Notas}

01. La acotación temporal elegida para esta investigación se centra en los 15 años en los que los dos socios fundadores del estudio, Kevin Roche y John Dinkeloo, trabajaron juntos, hasta la repentina muerte del último en junio de 1981.

02. La relación de las exposiciones que se han desarrollado en el MoMA desde 1929 hasta nuestros días está publicada y abierta al público en la página del museo. En los quince años que aquí se investigan se han contabilizado un total de 32 exposiciones dedicadas a la arquitectura. Ref. web 01: http://www.moma.org/learn/resources/ archives/archives_exhibition_history_list. Consultada el día 3 de junio de 2016.

03. La relación de obras incluidas en la exposición se ha encontrado en: The Museum of Modern Art Archives. Collection: PI. Series. Folder: II.B.648.

04. Nota de prensa emitida por el MoMA a los medios con la intención de publicitar la exposición. En esta misma nota se establecía un pase para la prensa el día 24 de septiembre de 1968 a las dos de la tarde, un día antes de la apertura al público. Lógicamente, el día 25 del mismo mes los periódicos daban cuenta de esta exposición, la mayoría de ellos, mencionando explícitamente la aportación de KRJDA a la muestra. Ref. web. 02: https://www.moma.org/d/c/ press_releases/W1siZilsljMyNjU4MCJdXQ. pdf?sha $=c 473970$ ca3ec5494. Consultada el 4 de abril de 2016.

05. "In addition to their architectural excellence, the examples chosen suggest an ambiance congenial to the immanent values of the collection and to the contemplative moments of the viewer". Palabras de Ludwig Glaeser en la nota de prensa enviada por el MoMA a los medios.

06. La relación de las obras expuestas y su división en distintos grupos según los conceptos que representaban se ha extraído de: The Museum of Modern Art Archives, NY. Collection: PI. Series. Folder: II.B.648.

07. Palabras de Ludwig Glaeser en la nota de prensa del MoMA a los medios. Ref. web. 02.

08. La otra imagen que incluye el artículo corresponde a la Nueva galería de Arte en Berlín, de Mies van der Rohe.

09. HUXTABLE, Ada Louise, "Architecture: A Museum is also Art, Exhibition shows", The New York Times, 25 de septiembre de 1968.
10. "The Art of Making the Magic Box", Progressive Architecture 11 (noviembre 1968): 56.

11. Nota de prensa enviada por el MoMA a los medios para ser publicada el 1 de octubre de 1970. Ref. web 03: https://www.moma.org/d/c/press releases/W1siZilsljMyNjcwNCJdX̄Q. pdf? sha $=$ c393818cff7547cc.

12. Extracto del cartel informativo de la entrada a la exposición, firmado por Arthur Drexler. Ref. web 03.

13. En esta exposición ya se evidenció que la figura de Kevin Roche funcionaba mejor en el ámbito mediático que la de John Dinkeloo (lo mismo podría decirse de Philip Johnson y John Burgee). El 14 de septiembre de 1970, y debido a la manifiesta preocupación de Kevin Roche, Arthur Drexler escribe una carta a John Dinkeloo, explicándole el motivo del nombre de la exposición, que no era otro que reducir el título de la misma. Es cierto que en el panel central de la exposición y en todos los textos que la acompañaban, por supuesto en el catálogo, aparece el nombre del estudio completo. The Museum of Modern Art Archives, NY, Collection: MoMA Exhs. Series. Folder: 940.11.

14. The Museum of Modern Art Archives, NY, Collection: MoMA Exhs. Series. Folder: 940.6.

15. Extracto de las cartelas que acompañaban las obras expuestas en la exposición y obtenida del archivo digital del MoMA. Ref. web 03

16. Extracto de las cartelas que acompañaban la exposición y obtenida del archivo digital del MoMA. Ref. web 03.

17. Extracto del cartel de la entrada a la exposición, firmado por Arthur Drexler. Ref. web 03.

18. Extracto del cartel inicial de la exposición, firmado por Arthur Drexler. Ref. web 03.

19. Obtenido de la lista de comprobación de material disponible en el archivo digital del MoMA. https://www.moma.org/d/c/ checklists/W1siZilsljMyNjcwMyJdXQ. pdf?sha $=9 \mathrm{c} 246 \mathrm{fcfce} 1522 \mathrm{ea}$

20. The Museum of Modern Art Archives, NY, Collection: MoMA Exhs. Series. Folder 940.12.
21. The Museum of Modern Art Archives, NY, Collection: MoMA Exhs. Series. Folder: 940.18.

22. The Museum of Modern Art Archives, NY, Collection: MoMA Exhs. Series. Folder: 940.6.

23. Palabras de Arthur Drexle entrevistando a Kevin Roche. The Museum of Modern Art Archives, NY, Collection: MoMA Exhs. Series. Folder: 940.6.

24. "Fotografías de más de 400 edificios, muchos de los cuales parecen rechazar los conceptos tradicionales de lo que es la arquitectura moderna, ilustran la reivindicación de la exposición de que la historia de la arquitectura moderna de las dos últimas décadas implica la elaboración de ideas propuestas por primera vez hace 30 o 40 años". Nota de prensa enviada por el MoMA a los medios. Ref. web 04: https://www.moma.org/d/c/ press_releases/W1siZilsljMyNzlwMSJdXQ. pdf?sha $=313626 \mathrm{~d} 5 \mathrm{cebd} 628 \mathrm{a}$.

25. Nota de prensa enviada por el MoMA a los medios. Ref. web. 04 


\section{Referencias bibliográficas}

- HUXTABLE, Ada Louise, "Architecture: A museum is Also Art, Exhibition Shows", The New York Times (25 de septiembre de 1968).

- "Creations of 3 Top Architects Shown", The New York Times (30 de septiembre de 1970).

-P.A. "The Art of Making the Magic Box", Progressive Architecture 11 (noviembre 1968): 56.

- PELKONEN, Eeva-Liisa. Kevin Roche: Architecture as environment, New Haven y Londres, Yale University Press, 2011.

-SMITH, C. Ray. “The Great Museum Debate", Progressive Architecture 12 (diciembre 1969): 76-85.

- STANISZEWSKI, Mary Anne, The Power of Display. A History of Exhibition Installations at the Museum of Modern Art. Cambridge, Londres: The MIT Press, 2003.

- Ref. web 01: http://www.moma.org/learn/ resources/archives/archives_exhibition_ history_list. Consultada el día 3 de junio de 2016.

- Ref. web. 02: https://www.moma.org/d/c/ press_releases/W1siZilsljMyNjU4MCJdXQ. pdf?sha $=c 473970$ ca3ec5494. Consultada el 4 de abril de 2016.

- Ref. web 03: https://www.moma.org/d/c/ press_releases/W1siZilsljMyNjcwNCJdXQ. pdf?sha $=c 393818 \mathrm{cff} 7547 \mathrm{cc}$.

- Ref. web 04: https://www.moma.org/d/c/ press_releases/W1siZilsljMyNzlwMSJdXQ. pdf?sha $=313626 \mathrm{~d} 5$ cebd628a. 